\title{
Transforming the Quality of Workforce in the Textile and Apparel Industry through Digital Empowerment (Working Paper)
}

\author{
Bessie CHONG \\ Director, Group Training and Talent Management Esquel Group, Hong Kong
}

\begin{abstract}
How to cite this paper: $\mathrm{CHONG}$, B. (2019). Transforming the Quality of Workforce in the Textile and Apparel Industry through Digital Empowerment (Working Paper). The Educational Review, USA, 3(8), 96-105. http://dx.doi.org/10.26855/er.2019.08.002
\end{abstract}

*Corresponding author: Bessie CHONG, Director, Group Training and Talent Management Esquel Group, Hong Kong.

Email: Chongbe@esquel.com

\begin{abstract}
Textile and apparel industry has long been stereotyped as "traditional" and "oldfashioned". As a non-traditional company in a traditional industry, Esquel encourages employees to innovate and to challenge the status quo. "You can code" campaign was initiated in 2015 to engage and propel staff at all levels towards its vision of "Making a Difference". The campaign aims to drive a sustained cultural transformation to turn the less technically minded employees into confident users of technology with computational thinking (CT) ability, through developing mobile apps. Developing mobile apps is a tool to empower the employees. By changing their attitude in using technology and equipping them with computation thinking ability, employees will become more systematic and innovative in solving problems at workplace, and eventually improve their productivity.
\end{abstract}

\section{Keywords}

Digital Empowerment, Problem Solving, Computational Thinking Education, App Inventor, Productivity

\section{Introduction}

Esquel believes that coding will soon become a basic job skill for everyone. It is vital for the employees to have some understanding of programming regardless of what professional they are in. Failing that, the career mobility of an individual may be hindered, and so as organizational growth. This is especially important for the manufacturing sector, as Industry 4.0 is fast approaching. The new age employees need to equip with a new set of skills. Founded in 1978, Esquel started as a shirt maker and have over the years developed the capacity to weave innovative technologies into its people-centric culture. With key production bases established in strategic locations in China, Malaysia, Vietnam, Mauritius and Sri Lanka, and a network of branches in the US, Europe and Asia, it exports over 120 million shirts per year and offer total shirt solutions to global apparel and textile markets, from concept to rack. Esquel employs 57,000 diversified workforce united under the corporate 5E culture - Ethics, Environment, Exploration, Excellence and Education, and the motto "Fun People Serving Happy Customers". It operates with an aspiration of "making a difference" by weaving positive impact to the employees, societies and environment.

\subsection{Business Challenges and Opportunities}

Esquel is in the industry of textile and apparel manufacturing, where all players face structural challenges, including rising labor and material costs, reduced profit margin and shortage of skilled labors. On the other hand, the rise of fast fashion further disrupts the industry by demanding quicker production cycles, more rapid prototyping and smaller order sizes. Therefore, the traditional manufacturing model with long lead time and mass production will no longer survive. The textile and apparel manufacturing industry employs over 75 million people worldwide (Stotz \& Kane, 2015) with an aggregate export amount over US\$744 billion in 2015 (World Trade Organization, 2015). The industry is still versatile, and has huge potential. The question is - how do manufacturers stay competitive and enable sustainable growth amidst the changing environment? Many players in this industry believe that growth must be tied with the overuse of labor and that competition must be based on low wages. Therefore, it is typical for those players to migrate the manufacturing base to chase for cheap labor. But Esquel decided to stay in locations where it has good operating conditions and to build local talent pool. Esquel strives to improve labor productivity 
to offset rising wages. It would rather improve the efficiency of the people and pay them well by integrating them into the technology, not by replacing them by using the technology. The coming fourth industrial revolution, known as Industry 4.0 , will provide Esquel with an opportunity to sustain its leadership position in apparel operations. The advent of the fourth industrial revolution is associated with the development of global industrial networks, to which all production processes of a wide variety of enterprises will be connected. As a result, computer interaction environment is developed around the modern human (Yastreb, 2015). That means employees would work with cyber-physical systems in a smart factory environment. "Combing human talent with technology empowerment enables people to do things humans are not equipped to do on their own," said Jeffrey Rayport, a professor of Harvard Business School and author of Best Face Forward. (Personnel Today 2017). For example, people can make use of the mobile technology and data to enhance real-time communication, improve alignment, and make timely decisions. Eventually, it will increase productivity and efficiency.

\subsection{People Challenges}

Nowadays, working environments are changing at unprecedented speed. The rise of robotics and artificial intelligence calls for new skills and competencies. In the workforce of Esquel, only 3.4\% have any technical qualifications, only $12 \%$ have a diploma or above, and 38\% were born before the personal computer became popular. There is a serious shortage of technically minded and savvy employees. It needs to turn those less technically minded employees into confident technology user. It needs to empower them to come up continuous improvement ideas to solve their own work problems systematically and independently. It needs to inspire the employees to participate in this transformation. It is a huge challenge as the target group is highly diversified, and spreads over 9 countries, 20 operations.

\section{2. "You Can Code 1.0" Campaign}

Coding is a skill that helps people learn how to think, systematically. By developing computational thinking, people can deconstruct problems step by step, and identify different recommendations. However, computational thinking may seem too abstract and coding may seem too technical. To engage everyone who does not have technical knowledge, a fun and practical approach is needed. The 'App Inventor' application developed by the Massachusetts Institute of Technology (MIT) was identified as the driver of this campaign.

The simple graphical interface of App Inventor allows an inexperienced user to create basic, fully functional mobile apps within an hour or less. It transforms the complex language of text-based coding into visual, drag-and-drop building blocks. It can change employees' perception of technology through this campaign. It would develop their logical reasoning skills, programming capabilities, and more importantly, computational thinking ability. Computational thinking is a fundamental skill for everyone, and it is a way humans solve problems (Wing, 2006). It includes problem decomposition, algorithmic thinking, abstraction, and automation (Yadav et al., 2017). By equipping computational thinking ability, employees will ultimately become innovators, problem-solvers, collaborators as well as process owners. They equip themselves for life. Yadav et al. further stressed given the irreplaceable role of computing in the working life of today, the competence to solve problems in technology-rich environments is of paramount importance.

"Therefore, there is a need to pay attention to CT as part of the broader concept of digital literacy in vocational education and training, as otherwise adults with only professional qualification may not be well prepared for the working life in the twentyfirst century". (Yadav et al., 2017, p.1065).

To Esquel, no matter whether they are workers, general staff, managers or executives, they need to have the attitude and ability to solve problems and realize ideas which will improve productivity.

\subsection{Champaign Design and Implementation}

This campaign is designed around how to change Attitudes, upgrade Skills and build Knowledge.

Table 1. ASK model

\begin{tabular}{|l|l|l|}
\hline To change & Attitude & $\begin{array}{l}\text { - by developing those ideas gradually from accepting, to understanding, to } \\
\text { embracing, to exploring } \\
\text { by showcasing employees that everyone can code }\end{array}$ \\
\hline To upgrade & Skills & $\begin{array}{l}\text { - by conducting workshops, activities, events and competitions } \\
\text { - by encouraging employees to innovate or to solve specific problems using the } \\
\text { technology }\end{array}$ \\
\hline To build & Knowledge & $\begin{array}{l}\text { - by developing an independent learning mindest } \\
\text { by creating a rich learning resource environment }\end{array}$ \\
\hline
\end{tabular}


"You Can Code 1.0" campaign started in 2015 to 2018, for 3 years. It is an awareness and experimental phase. It aims to create an awareness, engage the employees, promote computational thinking and have a taste of coding. The project team aspires to nurture the coding learning culture and make the program sustainable.

\subsection{Implementation of Phase 1 and Its Achievements}

In order to engage all employees, the role of IT throughout the campaign is purposely downplayed. The project team understands that it is impractical if only IT colleagues are involved in providing some classroom training and expect that employees will change the attitude towards technology. Therefore, an "all-in" approach was adopted and the campaign was launched out in 5 development stages.

Table 2. Five development stages and achievements

$\begin{array}{ll}\text { Stage } & \text { What have been achieved } \\ & \text { Workshops for senior management team and board members were conducted to collect their } \\ \text { feedback and get their buy-in. About } 90 \% \text { of them attended the training. Some of them also became } \\ \text { Esquel's pioneers. }\end{array}$
Some General Managers and Directors were invited to be the trainers to conduct workshops for Modelling other colleagues, from workers to managers. Almost 300 employees were trained. They reinforced the notion 'If I can code, you can code too'.

\section{Changing}

Cultivating

Realizing
43 super-users were identified and trained to be the change agents or ambassadors. They joined a customized master trainer course. Then they delivered training at different operations.

The master trainers were sponsored to launch a series of workshops and fun days, for the staff and for their kids in order to cultivate the skills and mindset. The ambassadors set up information and promotional booths to educate the frontline operators. A total of almost 800 people were trained.

The first "Esquel's App Challenge" Competition was organized in order to encourage the applications of the new skills. Many interesting and practical Apps were developed.

Through 28 workshops, over 2,430 training hours were provided for 1,200 participants, including 1,100 employees and 100 of their children from 10 locations in first 10 months. The strategy of teaching the children and letting children teach their parents back also worked well. Overall, the impact was encouraging. A lot of positive feedbacks were received -

- "Something looks complicated but can be very user friendly for us in building an App. Useful and valuable of some information/tools can be shared from the company!"- By a Sales Manager in Hong Kong

- "The introduction of the online programme 'App Inventor' is useful for non-professionals to build up our own app." - By a Senior Sales Executive in Hong Kong

- "Easy to operate for dummies. All ordinary people can participate in creating app without the support of IT". - by an Engineering Officer, in a Garment Factory

\subsection{Value Created from the Champaign}

The most important impact is the values created, including attitude change towards technology, employee engagement, employer branding, and process improvement. Many more app ideas from the employees were received. It is a belief after innovating once, employees are likely to innovate again. Now, non-IT employees can perform IT tasks, and even build prototypes by themselves. This in turn will allow IT professionals to focus on enterprise level app development.

Those applications help to save time and improve efficiency, while the broader benefits are incalculable. This campaign shows Esquel's commitment to upgrading their workers to become knowledgeable. It reinforces Esquel's brand as a caring and nontraditional company.

After the "You Can Code" campaign, it is found the rise of mobile app culture in Esquel. Employees are keen on thinking how to build some applications to improve some work-related or living-related issues. There is another interesting application, named Esquel Carpool, developed by the factory colleague using another software. The impact is enormous. 
Table 3. Some examples of the mobile apps developed in this campaign

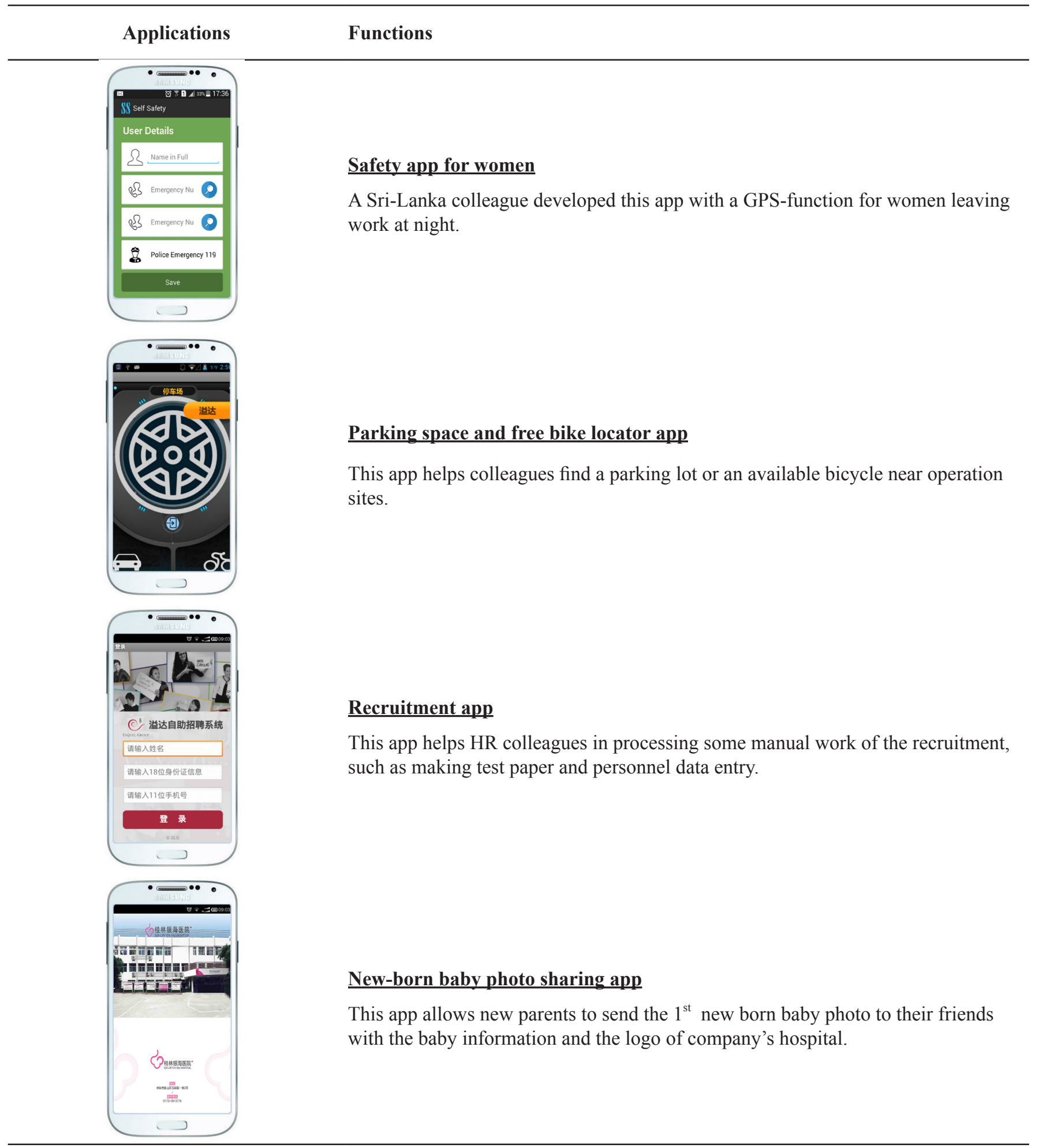

\subsection{The First Commercialized Mobile App in Esquel}

Esquel's largest operation is located in Gaoming, Foshan. It has about 23,000 employees working in several factories where are spread over the city of Gaoming. That means about $40 \%$ of Esquel employees are working and living here. Employees have to travel from home to these working locations, in similar timing, and similar routines every day. Many of them have to take company bus or city bus, and wait in a long line in sun, rain and wind. Commuting can easily take up half an hour or even one full hour per trip. For those 2,000 employees with their own private cars, the situation is no better. Driving to work is not at all pleasant when they have to be stuck in traffic and fight for the meagre 200 parking spaces available around the factories. A lot of times, they have to park far away and then take a 10-minute walk to the office. 
With this in mind, an employee in the factory initiated an idea to develop Esquel Carpool app to facilitate the carpool process in Esquel. Within the first 22 months, this App already recorded more than 75,000 carpools, with the saving of more than 57,000 litres of gasoline. It avoided the emission of 130.7 tons of carbon dioxide. This App changed their behaviour and inspired others to contribute in building a more sustainable lifestyle. More importantly, it provides a platform to make a connection with colleagues from different departments that promotes the caring culture. This software is free to any companies and organizations, and already make it available in the open source community GitHub.

\subsection{Impact on People - Story of a Garment Factory Worker}

Undeniably, many good apps idea and apps have been developed. However, the ultimate goal of this campaign is to change the attitude of employees in using technology and adopt a more systematic and innovative in solving problems. Employees' attitude and behavior change is important. It is found that in the process of developing their own mobile applications, the employees started to integrate computational thinking into their everyday work. They took attempt to analyze the problems by breaking them down and identifying the root cause, instead of jumping to quick fixes. The story of Yang Huamei illustrates how a basic coding training can bring an impact on a sewing worker.

Yang Huamei was a young woman from the southwest of China with an immense interest in fashion design and a desire to follow a career in apparel manufacturing. After graduating from high school in 2014, she joined Esquel as a sewing worker and brought many undeveloped fashion ideas that were waiting to be realized.

One day, Hua Mei found that the company was running a campaign. She believed that this campaign could teach her the computational skills necessary to turn her undeveloped fashion ideas to life; skills such as computational thinking, logical reasoning and simple programming. Without any hesitation, Hua Mei took the opportunity. Hua Mei and two other colleagues had built an app allowing users to mix- and-match the wardrobe.

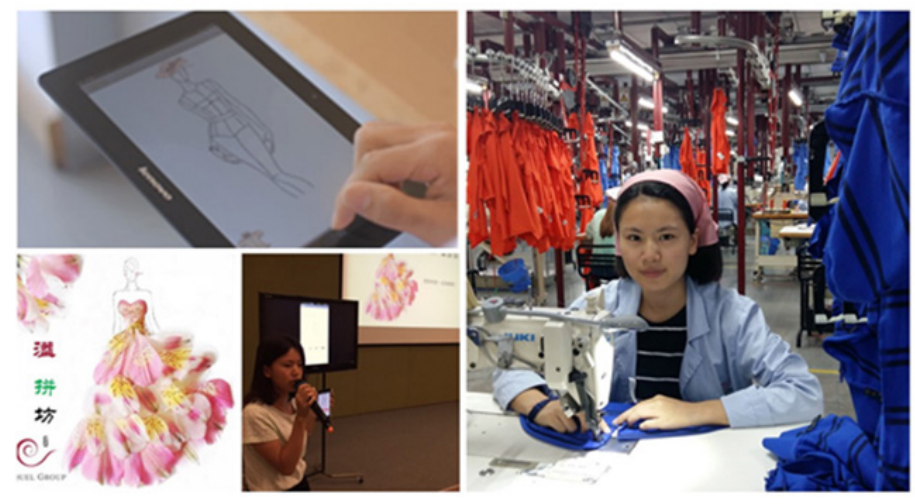

Figure 1. Yang Huamei and her teamwork

"Before joining 'You can code', I didn't even know what was meant by an 'app'! I have learned so much in the program, and now I appreciate the work of the technology gurus - however simple an app might seem, building one requires many steps and logical thinking!', said Hua Mei.

She also realized that the basic coding technique equips her with computational thinking ability, which in turn help her become an independent thinker. As a sewing worker, from time to time, she faced problems in operating her sewing machine and managing the sewing quality. Before she learnt coding, whenever she came across problems, she simply asked the mechanic to fix it or change some machine parts by herself. She would not bother to understand the problems, identify the root causes, and think about how to prevent them in future. But now, she becomes proactive in learning technical skills and integrating the computational thinking ability to solve her everyday work problems. She also aspires to evolve from a sewing worker to a technician one day.

\subsection{Learning Momentum - Campaign Evolution}

Hua Mei's story is one of many at Esquel that inspires many others to learn the technique of app coding. With the initial success during the first ten months, the project team committed to carry on this transformational journey and take one step further from creating a simple mobile application to developing a stimulated real-life application in workplace. It started to teach colleagues essential skills to build a microcontroller-based embedded system which is monitored by a mobile app developed by App Inventor. It is because Esquel has many "Internet of Things" applications. For example, auto-guided vehicles and drones are adopted in a factory for transportation use. By learning the logic behind those basic programming techniques with the connectivity with the physical devises as an example, colleagues can replicate the setting and methodology to create their own IoT or Arduino prototypes. Once colleagues figure out most of the intelligent production and process systems found in the work environment, in fact, are programmed by the basic computer logic, they will have confident in making recommendations and asking questions on the current practices and systems. 


\subsection{You Can Code 1-2-3}

To continue the coding learning journey, a progressive and systematic learning path was designed. Three levels of workshops, according to the difficulty and complexity of the App, were organized (Figure 2).

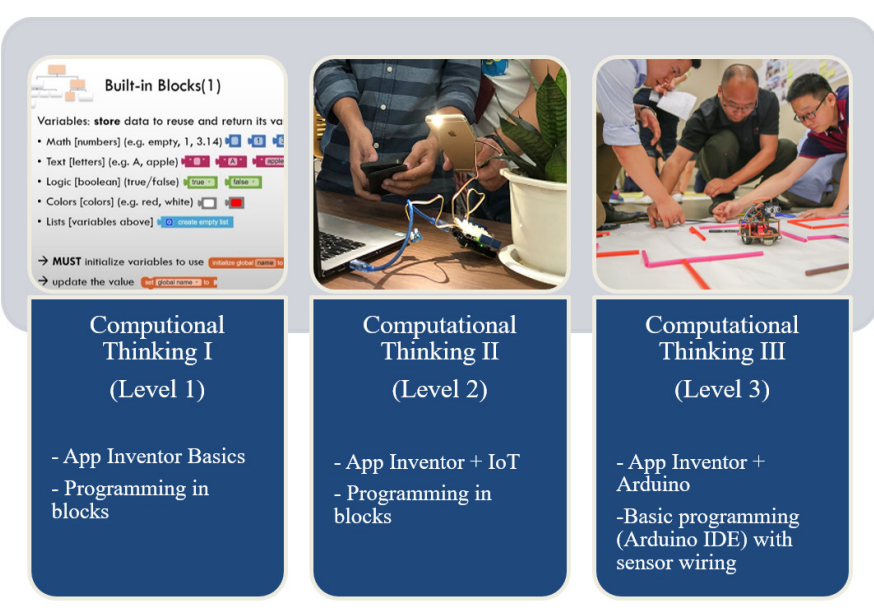

Figure 2. You Can Code 1-2-3 Workshop

Level 1 workshop focuses on basic App Inventor techniques, it serves as a beginner course. It aims to help new participants equip with the basic skills, and also help to refresh the previous practice. At this level, not all block building instructions are given, except basic functions and interface of the App. Some instructions are deliberately left blank, and only partial block building instructions are provided. Participants need to complete the instructions and build the blocks according to their needs and understanding. Some custom-made design of the interface and functions are recommended to demonstrate their creativity, the comprehensiveness of logic, and the ability of computational thinking.

Level 2 workshop focuses on programming techniques applications on a micro-ecosystem. Participants are required to develop a Smart Plantation Monitoring System based on IoT to monitor a small plant growth. The mobile app collects humidity and light data from the plant via a sensor node. Participants base on the data collected to build a decision-making mechanism. With the combination of wireless sensor network, embedded development and data transmission, colleagues can receive recommendation instantly, such as pouring water or giving more light in order to keep the plant healthier. This device set-up demonstrates how the computer programming would aid decision making.

Level 3 workshop focuses on providing a simulated operation environment for problem solving. Participants are taught how to program the Arduino to perform certain tasks, such as turning on the lights, making a device turns, moving forward and backward, and escaping from a maze. The set-up simulates the auto-guided vehicle operating in the factory floor for moving raw materials, semi-products and products.

To implement a series of workshops to different locations, more ambassadors and change agents are identified. Colleagues, particularly the young generation, are invited to join the master training. Esquel also incorporated the basic App Inventor training into their management trainee training curriculum. Management trainees (MTs) spread across different locations were trained and will take ownership in fostering the learning culture by teaching back to colleagues at their own locations.

\subsection{Cycles of Learning}

The project team strongly believed peer learning is more effective in promoting the use of technology and overcome their fear of technology adoption. More local needs on improving work-related or life-related issues can be solved by themselves.

During the period of 2016-2018, about 30 more "hour of code" workshops were conducted and 700 colleagues more (including those management trainees, ambassadors and local trainers) were trained. But the whole learning progress is slower than what the project team expected. It is found that only $20 \%$ of local trainers are active trainers and delivered 1-2 workshop locally in 2 years.

The project team identified the following key challenges and issues of "You Can Code 1.0" Campaign:

1. The training adoption rate is still low and varied across different locations because the digital literacy of employees across different locations is varied significantly. Employees in Guangdong area, especially in our largest operation, are more proactive in learning coding (Appendix 1). This would be because the operation is the largest one and more mature. More employees are more ready to change and aspire to be upgraded. Compared to other operations, especially those overseas operations, classes were delivered by corporate trainers, not local trainers.

2. Workers should be the main target training group but the program reachability to them is still low (Appendix 2). Only 
about $0.1 \%$ of workers had joined the workshop. This is the biggest pool of workforce in the Company and low percentage means the critical mass has not been reached. If this is the case, it will become an obstacle of the transformational journey.

3. Business impact on productivity improvement has yet to show, that may affect the motivation of local management team and participants. Even though some great mobile apps ideas and apps were developed, the local management team and colleagues may not see the immediate business values. Compared to those technical production training, the priority of "You Can Code" workshop is low. The local teams prefer to drive the production rather than long-term people development, whereas time investment and effort in cultivating coding culture and CT ability is huge but the business impact takes time to realize.

Therefore, even the satisfaction rate of "You Can Code" (hour of code) workshop is high, over 9/10 in average, the scalability is limited.

\section{3. "You Can Code 2.0" Campaign}

The project team reviewed the whole approach and decided to provide more corporate resources and propose a more focused and customized approach. The training impact on the business will also be measured systematically. Therefore, another 3-year program plan is developed.

In the "You Can Code 2.0" campaign, worker level will become the main target group. The project team aims to increase their confidence of using technology through digital empowerment. Besides App Inventor, other digital tools will be used to engage the workers according to their digital readiness and engagement levels.

Since it is expected that the potential participant group is huge (around a few thousands) and the engagement activities are continuous with high flexibility and practicality. The requirements on the quality of the ambassadors and local trainers become high too. Therefore, a structured, continuous, and long-term development is needed in order to engage them and train them up to the standard. Riding on the concept of Key Opinion Leaders (KOL), the project team aims to recruit a group of capable and committed KOL to support this campaign. A robust recruitment process, through General Managers' nomination and self-application from the talent group, has been conducted. Selection test will be applied to screen and categorize the KOL in different competency levels for different roles and responsibilities, including learning booth promotion, training program design and delivery, hackathon support, hotline support, etc.

In order to get the buy-in from the management team, the project team understands the important of creating more successful stories, like Yang Huamei, and showing the business values of computational thinking at workplace. A systematic measurement on the program impact is needed. There are three levels of measurements:

Table 4. Learning Impact Measurements

\begin{tabular}{|l|l|}
\hline \multirow{2}{*}{ Level 1} & $\begin{array}{l}\text { Program Level - Program Coverage, Success Rate of App Creation and Satisfaction } \\
\text { Rate of Learning, such as } \\
\text { - Number of workers joined and successfully completed the tasks in the workshops } \\
\text { - Number of workshops and the satisfactory level } \\
\text { - Number of KOL (master trainers and ambassadors) trained }\end{array}$ \\
\hline Level 2 & $\begin{array}{l}\text { Attitude and Behavioral Change on Technology, such as } \\
\text { - Any changes on pre and post workshop self-evaluation on the attitude towards technology } \\
\text { - Any changes on pre and post workshop ability test of performing a task via tablet/ } \\
\text { computer on their own or solving some problems }\end{array}$ \\
\hline Level 3 & $\begin{array}{l}\text { Business Impacts, such as } \\
\text { - Any changes on engagement, competency, and productivity } \\
\text { - Anymer of app created and their potential values }\end{array}$ \\
\hline
\end{tabular}

\subsection{Literature Review}

\subsubsection{Digital Empowerment}

People feel empowered if they can have more accessibility and control. Digital empowerment means giving people new 
abilities and ways to participate and express themselves through digital technology (Makinen, M, 2006). According to the report of Personnel Today (April 2019), the frontline workers, who spend most of their time making products or managing operations, have too often been left at the back of the line when it comes to digital transformation. The use of tablets, smartphones and virtual workspaces can empower and enable frontline workers.

Therefore, business leaders start to extend the digital efforts to different job holders to increase productivity and efficiency, improve quality of product or services, increase innovation and employee satisfaction.

\subsubsection{Computational Thinking and Digital Empowerment}

Tissenaum, M. et al, (2017) stated that development of computational thinking (CT) is particularly critical in supporting individual growth. From CT perspective, digital empowerment describes the recognition of their capacity can have active, positive roles creating tools and artifacts with computer to enhance their lives and the lives of people around them. They believed the true empowerment comes from creating interventions that move into the physical world - interventions that allow learner to analyze problems in their communities and devise real solutions to these problems. Thus, digital empowerment should bring positive impact on the problem-solving ability. Teaching coding is not to teach people how to code, rather than to encourage them to learn to think computationally to address problems around them.

\subsection{Business Values and Objectives}

This research enables the Company to explore some key relationships on digital empowerment and business values. Besides App Inventor, different digital tools and experiential learning will be used to engage the employees. The following business questions can be addressed in this research:

- What are the relationships between digital exposure, attitude change, ability improvement, engagement level and productivity change?

- What will be the effective tools to help employees change the attitude of using technology?

- What will be the effective tools to develop computational thinking/ problem solving ability?

- What will be the effective tools to engage employees?

- How much of different digital exposures should be provided in order to demonstrate positive impact on the confidence of using technology, problem solving, engagement, and productivity improvement?

- What kinds of digital exposures/ experiences will contribute the most on positive impact?

- Can the digitally empowered employees help boost the productivity?

The findings will help the Company develop an effective digital strategy for people development and employee engagement. Eventually, it will help the Company to build a strong company brand and increase its organizational capability in long-run.

\subsection{Assumption and Hypotheses}

The project team aims to transforming the quality of workforce in the Company through digital empowerment. Digital empowerment is assumed to make the workforce more engaged and improve the quality of workforce, especially of those who lack of technology exposure/ STEM education. By increasing the confidence of using technology and computational thinking ability, the workforce will become positive on changes and proactive in initiating improvement actions. Eventually, their engagement level, productivity, efficiency or competency will be improved.

App Inventor will be the main driver of digital empowerment tool because App Inventor is widely used in computational thinking for young generation and is shown it can help to develop computational thinking of the users and initiate computational actions (Tissenbaum, M. et al., 2019).

\section{The hypotheses:}

1. Employees with digital exposure (empowerment) will have more confidence in using technology than employees without empowerment.

2. Employees with digital exposure (empowerment) will have higher engagement level than employees without empowerment.

3. Employees with exposure (empowerment) are more competent in problem solving than employees without digital empowerment.

4. The more the digital exposure (empowerment), the more confidence in using technology, and the higher the productivity.

5. The more the digital exposure (empowerment), the higher the engagement level, and the higher the productivity.

6. The more the digital exposure (empowerment), the more competent in problem solving, and the higher the productivity.

\subsection{Conceptual Framework}




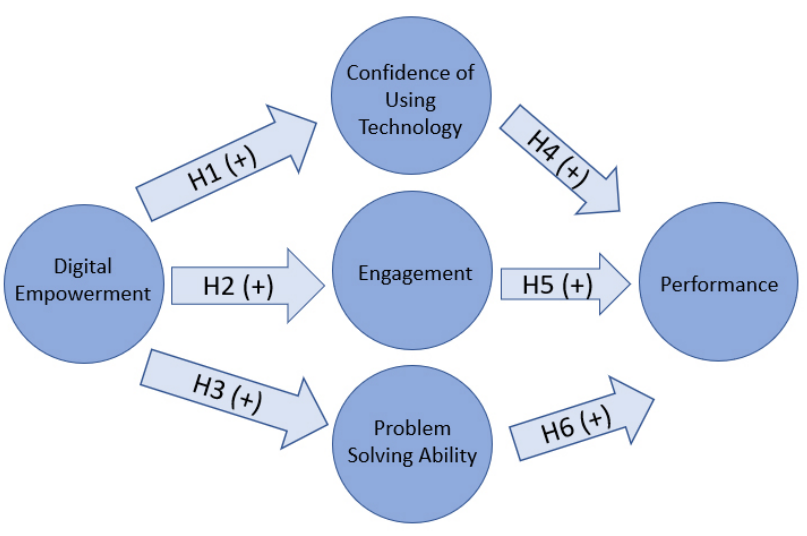

Figure 3. Conceptual Framework

\section{Definition:}

- Digital Empowerment will be measured by the level of formal training for CT development and informal learning for digital involvement. A series of digital experiential learning activities will be provided, including a hour of code "App Inventor" workshop, modular-based "I Can Code" certification program, learning booth, Hackathon, and App Challenge Competition, etc.

- Confidence of using technology refers to attitude of not afraid of using technology. It can be measured by the pre-survey or ability to perform certain tasks on digital tools. The project team proposed to use the scale of "Affinity for Technology" and “Technophobia" level (Edison, S. \& Geissler, G. (2003)) to measure the participants' attitudes towards technology.

- Engagement refers to employee engagement level. It can be measured by retention rate, satisfaction rate, etc.

- Problem solving ability is used to represent computational thinking ability. It can be measured by the ability of completed certain tasks/assignments or test results. It can also be measured by their self-rating on "Need for Cognition" and "Selfefficacy" (Edison, S. \& Geissler, G. (2003)) before and after digital empowerment.

- Performance refers to employee performance. It would be measured by quality of work, productivity and efficiency (for workers), and performance appraisal (for staff).

\subsection{Research Methodology}

The project team will conduct A/B Test to compare employees with digital empowerment and without (or very little) digital empowerment. A group of workers (Group A) will be invited regularly to join a series of digital experiential learning activities (including formal and informal) throughout 2 years. The project team will monitor their progress - ability and confidence of using technology while they visit those learning booths and join workshops. Their learning progress (ability changes) with their engagement level, efficiency and competency changes will be measured and compared against those who do not participate or just participate very few digital experiential learning activities (Group B). In addition, the project team will compare the engagement level, work efficiency and competency of Group A, before and after training to see their changes in 2 years.

Regression model is used to measure the effects of different variables of digital empowerment that potentially have an impact on the confidence of using technology, problem solving ability, engagement level and productivity.

\section{Conclusion}

This research is the first attempt to measure the impact of computational thinking ability on adults in a traditional industry. The project team aims to provide various digital learning experiences to enrich the digital exposure of the workers and eventually to change their attitude of using technology. It is to believe once the attitude changes, their behaviour will be changed positively towards technology. They will become more open in using the technology. They can also adopt a more systematic way to think and solve a problem. They will become more positive when encountering problems and proactively propose innovative ways to solve problems at workplace. Digital empowerment is a signal to the employees that they can come up with great initiatives to make the Company a better work environment, and as a result, the employee engagement can be enhanced. The stable and skilled workforce can drive a higher productivity and enable the Company to sustain and lead in this fierce competitive business environment and disruptive industry 4.0 era.

\section{Appendix}




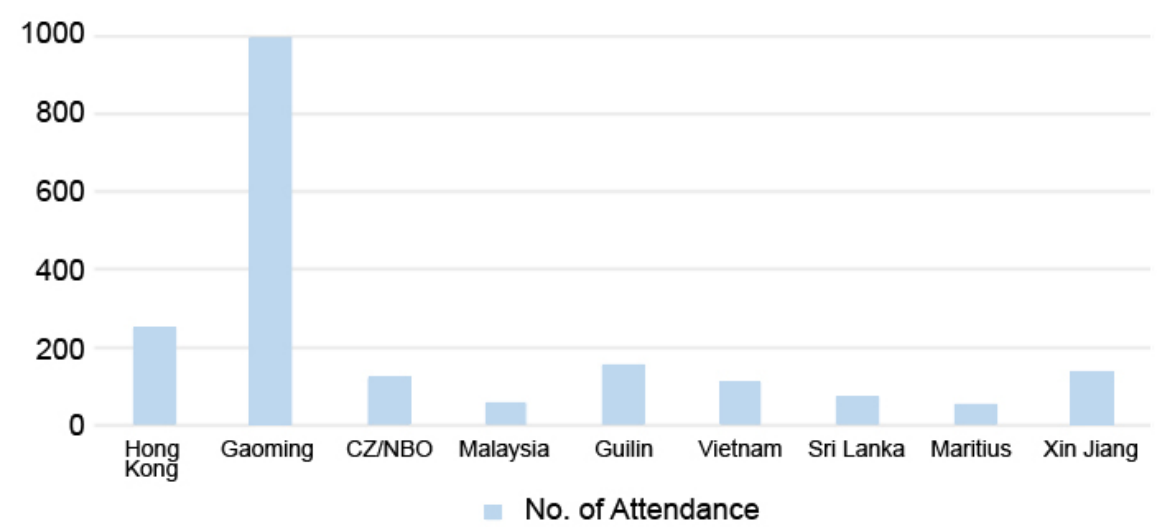

Appendix 1. Training Adoption Across Different Locations

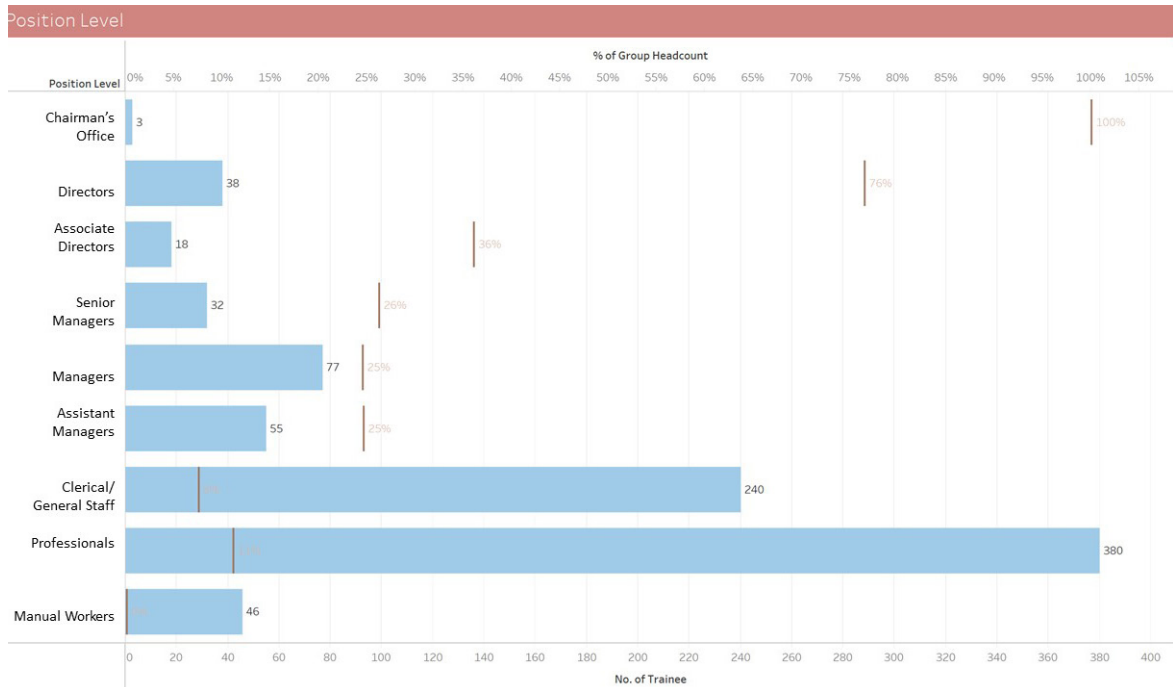

Appendix 2. Training Adoption Across Different Position Levels

\section{References}

Edison, S. \& Geissler, G. (2003). Measuign Attitude Towards General Technology: Antecedents, Hypotheses, and Scale Development. Journal of Targeting, Measurement and Analysis for Marketing, 12(2), 137-156

Makinen, Maarit (2006). Digital Empowement as a Process for Enhancing Citizens' Participation. E-Learning, 3(3), 381-395.

Peronnel Today (2019, April). Don’t Exclude Frontline Workers from Your Digital Vision. Retrieved from https://www. personneltoday.com/hr/dont-exclude-frontline-workers-from-your-digital-vision/

Stotz, L. \& Kane, G. (2015, February). Facts on The Global Garment Industry. Clean Clothes Organization. Retrieved from https://cleanclothes.org/resources/publications/factsheets/general-factsheet-garment-industry-february-2015.pdf

Tissenbaum, M. et al. (2017). Critical Computational Empowerment: Engaging Youth as Shapers of The Digital Future. Global Engineering Education Conference (EDUCON), 2017 IEEE, 1705-1708.

Tissenbaum, M. et al. (2019). From Computational Thinking to Computational Action. Communications of the ACM, 62(3), 34-36. Wing, J. M. (2006). Computational Thinking. Communications of the ACM, 49(3), 33-35.

World Trade Organization (2015). World Trade Organization. Retrieved from http://stat.wto.org/StatisticalProgram/ WSDBViewData.aspx? Language $=\mathrm{E}$

Yadav, A. et al. (2017, January). Computational Thinking as an Emerging Competence. Research Gate. Retrieved from https:// www.researchgate.net/publication/307942866

Yastreb, N. (2015). The internet of things and the forth industrial revolution: the problem of humanitarian expertise. Journal of Scientific Research and Development, 2(8), 24-28. 